\title{
Recombinant production of growth factors for application in cell culture
}

\author{
Meenakshi Venkatesan ${ }^{1 \#}$, Cameron Semper ${ }^{2 \#}$, Rosa DiLeo $^{1}$, Nathalie Mesa $^{1}$, Peter J Stogios ${ }^{1 *}$, \\ and Alexei Savchenko ${ }^{1,2^{*}}$
}

${ }^{1}$ Department of Chemical Engineering and Applied Chemistry, University of Toronto, Toronto, Ontario, Canada M5S 3E8

${ }^{2}$ Department of Microbiology, Immunology and Infectious Diseases, University of Calgary, Calgary, Alberta, Canada T2N 4N1

${ }^{\#}$ These authors contributed equally

*Corresponding authors

p.stogios@utoronto.ca

alexei.savchenko@ucalgary.ca 


\section{Summary}

2 Culturing eukaryotic cells has widespread applications in research and industry, including the

3 emerging field of cell-cultured meat production colloquially referred to as "cellular agriculture".

4 These applications are often restricted by the high cost of growth medium necessary for cell

5 growth. Mitogenic protein growth factors (GFs) are essential components of growth medium and

6 account for upwards of $90 \%$ of the total costs. Here, we present a set of expression constructs

7 and a simplified protocol for recombinant production of functionally active GFs, including FGF-

8 2, IGF-1, PDGF-BB and TGF- $\beta 1$ in Escherichia coli. Using this expression system, we produced

9 soluble GFs from species including bovine, chicken, and fish. Bioactivity analysis revealed

10 orthologs with improved performance compared to commercially available alternatives. We

11 estimated that the production cost of GFs using our methodology will significantly reduce the

12 cost of cell culture medium, facilitating low-cost protocols tailored for cultured meat production

13 and tissue engineering. 


\section{Introduction}

Cellular agriculture is a rapidly emerging field that seeks to leverage biotechnology to

27 produce agricultural products from cell cultures (Post et al., 2020). Study in cellular agriculture

28 has intensified in recent years due to the proposed environmental and ethical benefits compared

29 to traditional agriculture (Mattick, Landis, Allenby, \& Genovese, 2015). The field has spurred

30 the development of a range of products (e.g., milk produced using recombinantly-produced

31 protein (Geistlinger, 2017)); however, the primary focus of research in cellular agriculture relates

32 to the development of cell-cultured meat (CCM). Also referred to as cell-based meat or

33 cultivated meat, these products marry cell culture, tissue engineering, and bioprocessing to

34 develop three-dimensional "slab" meat products independent of animals (save for the initial

35 biopsy used for cell line development or primary cell isolation) (Melzener, Verzijden, Buijs,

36 Post, \& Flack, 2021). Direct comparison between CCM and traditionally produced meat products

37 predict that an animal-free approach to meat production can reduce greenhouse gas emissions by

38 up to $95 \%$, conserve land and water usage, attenuate the overuse of antibiotics in agricultural

39 settings, and reduce the incidence of zoonotic infections (Godfray et al., 2018). Furthermore, the

40 development of CCM as a bona fide food source has the potential to decentralize supply chains

41 and address issues related to food insecurity and sustainability. The potential benefits of CCM

42 have been well studied; however, for these advantages to be actualized there are several

43 technological challenges that continue to remain as significant hurdles.

The core technology at the foundation of CCM production is eukaryotic cell culture. Cell

45 lines or primary cells of bovine, avian, and teleost origin are used to produce cell-cultured beef,

46 chicken, and fish, respectively. Within the field of cellular agriculture, and eukaryotic cell

47 culture more broadly, cells are commonly supported with the addition of 5-20\% fetal bovine 
serum (FBS). FBS is obtained from blood and used as a growth supplement to sustain in vitro

49 cell culture by providing hormones, lipids, micronutrients, and, most critically, mitogenic growth

50 factors (GFs) that promote cell division (van der Valk et al., 2018). The use of FBS is near-

51 ubiquitous in research settings; however, its continued use within cellular agriculture is seen as

52 problematic for a number of reasons. FBS is costly, it can suffer from batch-to-batch variation

53 that can result in issues of reproducibility or reduced yield, and the fact that it is sourced from

54 fetal calves undermines the ethical advantage afforded by CCM compared to meat produced

55 using traditional agriculture.

56 Serum-free alternatives to FBS have been commercially available for decades and in the

57 case of chemically defined serum-free media (SFM), they offer improved consistency more

58 compatible with the controlled manufacturing processes adopted in CCM production.

59 Commercially available serum-free options include Essential 8 (Chen et al., 2011), TeSR, and

60 FBM and recent efforts have endeavoured to use these as templates for creating low-cost serum-

61 free formulations tailored for iPSC cells (Kuo et al., 2020) and bovine satellite cells (Stout,

62 Mirliani, White, Yuen, \& Kaplan, 2021). While SFM removes the ethical concerns related with

63 the use of FBS and offers improved consistency in cell culture performance, it is even more cost

64 prohibitive than FBS-containing media. At current prices, the use of SFM for the production of

65 CCM creates a significant barrier for competitive entry into consumer markets.

Mitogenic GFs, such as basic fibroblast growth factor (FGF-2) and transforming growth

67 factor $\beta 1$ (TGF- $\beta 1$ ), are the main cost drivers of SFM, estimated to contribute more than $95 \%$ of

68 the total cost (Specht, 2020). These signalling proteins bind to cell surface receptors activating

69 several downstream pathways to result in cell proliferation, cell migration, differentiation, and

70 apoptosis, and are added as a supplement to SFM to mimic the GF composition provided by 
71 traditional serum additives. While a wide array of GFs are used to support eukaryotic cell

72 culture, many of which are tissue/cell-type specific, there are a subset which are considered

73 essential for supporting cell proliferation in general (Yao \& Asayama, 2017). These GFs are key

74 components of SFM used to support cell growth for academic and cellular agriculture

75 applications.

high cost. They are of eukaryotic origin and typically require some level of post-translational modification, such as oxidation or glycosylation, and proper disulfide bond formation is essential for protein folding and maturation into their biologically active conformation. Consequently, production of these proteins in bacterial expression systems has been challenging. Strategies to

81 address this issue include refolding GFs expressed in bacteria from inclusion bodies or using 82 mammalian cell culture systems (e.g., CHO, HEK293) for their production (Alexander, Hesson,

83 Mannarino, Cable, \& Dalie, 1992; Zou \& Sun, 2004). Both strategies result in high costs and

84 render SFM cost-prohibitive for use in the production of CCM (Tripathi \& Shrivastava, 2019).

86 compatible with a target protein, offers advantages in terms of speed, tractability, and cost

87 (Rosano \& Ceccarelli, 2014). Several advancements in strain engineering have made E. coli an

88 improved host for production of disulfide-containing proteins. Mutation of $\operatorname{tr} x B$ and gor genes

89 (Bessette, Aslund, Beckwith, \& Georgiou, 1999), as well as cytoplasmic overexpression of

90 disulfide bond isomerases (e.g., DsbC) (Lobstein et al., 2012) are strategies that have facilitated

91 the expression of disulfide-containing proteins in E. coli, culminating in the development of

92 commercial strains such as Rosetta Gami B (Novagen) and SHuffle T7 (NEB), respectively.

93 Additionally, the use of redox fusion partners such as thioredoxin (Trx) (LaVallie et al., 1993), 
94 glutathione S-transferase (GST) (Schafer, Seip, Maertens, Block, \& Kubicek, 2015), and

95 disulfide bond isomerases (DsbA, DsbC) (Nozach et al., 2013) have greatly improved soluble

96 expression of proteins that contain disulfide bonds in E. coli. Despite these advancements, the

97 use of E. coli to produce soluble mitogenic GFs remains underexplored as commercial

98 production still relies on refolding in instances where $E$. coli is the chosen expression host.

99 To facilitate cost reductions in SFM and to advance its adoption in the field of cellular

100 agriculture, we screened a myriad of GF genes, fusion partner combinations and E. coli strains to

101 develop a collection of expression systems that allow for production of biologically active

102 mitogenic GFs in E. coli. As a result, we were able to identify expression conditions to

103 successfully produce several GFs from a variety of species including those useful in CCM

104 production (bovine, avian, fish). Expressed GFs can be purified using a one-step immobilized

105 metal affinity chromatography (IMAC) purification procedure with an optional additional

106 “clean-up" step. This methodology is feasible for academic labs as well as CCM start-up

107 ventures and results in biologically active GFs able to promote cellular proliferation. Using this

108 methodology, we identified GF orthologs that provide higher potency than commercially

109 available counterparts. Finally, we provide a techno-economic assessment to show that our

110 methodology facilitates significant cost reductions associated with the recombinant production of

111 mitogenic GFs. These results have the potential to significantly reduce the cost of SFM

112 formulations in the field of cellular agriculture and eukaryotic cell culture applications more

113 broadly.

\section{$114 \underline{\text { Results }}$}

\section{Selection of growth factors and expression system components}


GFs to target for cost effective production were selected based on their prevalence in FBS

117 or in current SFM-based formulations, and their likelihood of being widely adopted in CCM

118 production. Based on these criteria, we focused on four major GF families represented by

119 fibroblast growth factor (FGF-1 and FGF-2), platelet-derived growth factor (PDGF-BB), insulin-

120 like growth factor (IGF-1 and IGF-2) and transforming growth factor beta 1 (TGF- $\beta 1$ ). For each

121 of these families we selected multiple representatives from diverse species within available

122 genome sequences to sample sequence diversity and to expand the repertoire of GFs able to

123 support the growth of cells relevant for cultured meat production. Furthermore, we tested our

124 most successful expression strategies on additional GFs including leukemia inhibitory factor

125 (LIF), epidermal growth factor (EGF) and myostatin/growth differentiation factor 8

126 (MSTN/GDF8).

127 We chose to express the GFs in E. coli, which remains the most widely used, versatile,

128 and inexpensive recombinant expression system. Our selection of specific E. coli strains and

129 expression vectors was driven by the general goal of maximising the production of GFs in a

130 soluble and biologically active form that would also allow for simplified purification using a

131 standard IMAC protocol. Considering the prevalence of intramolecular disulfide bonds in many

132 GF proteins, we selected commercially available E. coli strains altered to accommodate the

133 expression of such proteins including Rosetta Gami 2(DE3) (Novagen), Origami B(DE3)

134 (Novagen) and SHuffle T7 express (NEB) in addition to BL21-Gold (DE3) as the hosts to test

135 for recombinant GF production.

136 For our first attempt, each of the selected GFs was expressed along with an N-terminal

137 polyhistidine (6xHis) tag or the classic redox fusion partner, thioredoxin A (TrxA). To assess the

138 success of these protein production strategies we performed small-scale expression tests of 
proteins for each family of GF in the selected E. coli host strains and screened for the presence of

140 soluble intracellular protein expression using SDS-PAGE (Figure 1A). We achieved limited

141 success with these two fusion partners for some GF families, so we expanded our screen to

142 include additional redox and non-redox fusion partner sequences. For non-redox fusion partners,

143 we used the 97 amino acid SUMO ubiquitin-like protein from Sacchromyces cerevisiae

144 (Addgene plasmid \#29711) and the B1 domain of the Streptococcal protein G (GB1) (Cheng \&

145 Patel, 2004), the latter of which has been successfully used to facilitate soluble expression of

146 human EGF in E. coli (Zheng, 2016).

147 For additional redox fusion partners, we incorporated disulfide bond isomerases (E. coli

148 DsbA or DsbC) that promote formation of disulfide bonds and have been previously described as

149 potent facilitators of soluble expression of disulfide-bond rich proteins in E. coli. For this, we

150 modified a previously constructed T7-based expression vector, pMCSG53 (Biancucci et al.,

151 2017; Eschenfeldt et al., 2013), to include either the leaderless DsbA or DsbC protein as an N-

152 terminal fusion partner for the GF protein. These new vectors, pMCSG53-DsbA and pMCSG53-

153 DsbC, are LIC and Gibson Assembly compatible, allowing for high-throughput entry of GF

154 sequences to promote investigation of amino acid sequence diversity within these protein

155 families. All expression constructs included a Tobacco Etch Virus (TEV) protease cleavage site

156 (ENLYFQG) between the tag and GF sequence to allow for removal of the fusion partner after

157 purification.

The expression level of GFs was tested on a small scale (see Material and Methods for

159 details) to identify the most promising strain and construct combinations for soluble recombinant

160 GF expression (Figure 1A). An overview of the most successful expression combination(s) for 
161 each GF is summarized in Figure 1B. Below we discuss the outcome of these strategies for each

162 of the selected growth factor families in detail.

163 Expression and purification strategy for fibroblast growth factor (FGF-1 and FGF-2)

164 FGF-1 (acidic FGF) and FGF-2 (basic FGF) are 155 amino acid proteins that exert

165 mitogenic and angiogenic activities on target cells through interaction with fibroblast growth

166 factor receptor (FGFR) and subsequent activation of the downstream RAS-MAPK, P13K-AKT,

167 STAT, and PLC $\gamma$ signalling pathways (Brewer, Mazot, \& Soriano, 2016). FGF-2 also plays a

168 role in tissue development and repair by binding to heparin sulphate cofactor (Koledova et al.,

169 2019). FGF is a critical component of cell culture medium, particularly for maintaining

170 pluripotency of stem cells and their proliferative capacity (Mossahebi-Mohammadi, Quan,

171 Zhang, \& Li, 2020). It is also a major mitogen in FBS, required for proliferative activity of cells

172 and is typically present in the range of 10 to $100 \mathrm{ng} / \mathrm{mL}$ in cell culture medium (Yang \& Xiong,

173 2012).

174

Both FGF-1 and FGF-2 have been shown to be prone to proteolytic degradation and

175 denaturation in cell culture, contributing to their relatively short half-life and present a major cost

176 driver for cell culture medium. These undesirable properties can be mitigated through addition of

177 heparin to the growth medium; however, this strategy adds to the overall medium costs and may

178 not meet food safety requirements for CCM applications. FGF variants offering improved

179 thermostability have been identified through protein engineering efforts (Benington, Rajan,

180 Locher, \& Lim, 2020; Zakrzewska, Krowarsch, Wiedlocha, \& Otlewski, 2004), but their

181 approval for application in CCM products may be complicated due to their "genetically-

182 modified" categorization. With these shortcomings of currently available FGF in mind, we 
183 developed a workflow to allow for low-cost bacterial expression and purification of an array of

184 FGF orthologs.

FGF-1 and FGF-2 have been previously produced recombinantly in E. coli with a variety

186 of fusion partners (e.g., TrxA, 6HFh8, (Gasparian et al., 2009; Kim et al., 2021)); however, these

187 approaches often require multiple purification steps and the use of specialized chromatography

188 equipment. In contrast, we tested the production of these GFs by a single step IMAC protocol,

189 followed by a TEV protease tag cleavage and removal using the same affinity resin used for

190 initial purification as shown in Figure 2a (see details in Materials and Methods). Additional

191 modifications in our methodology from previous expression strategies for FGF proteins include

192 the use of the synthetic codon-optimized genes and sampling of 21 sequence orthologs (5 FGF-1,

19316 FGF-2) to capture a representative level of amino acid sequence diversity within this protein

194 family. Our strategy allowed for successful expression and purification of 5 FGF-1 and 16 FGF-

1952 from terrestrial (bovine, chicken, sheep, human) and aquatic (Atlantic salmon, Nile tilapia,

196 Chinook salmon) species (Tables S1 \& S2). The protein yields of purified FGF-2 ranged from 7

$197 \mathrm{mg} / \mathrm{L}$ for Bos taurus (bovine) to $36 \mathrm{mg} / \mathrm{L}$ for Oryzias latipes (rice fish), highlighting significant

198 variation in FGF ortholog yield while expressed under the same general conditions (Figure S1).

199 Expression and purification strategy for Platelet-derived growth factor (PDGF-BB)

$200 \quad$ Platelet-derived growth factors (PDGF) are potent mitogens for a variety of cells

201 including fibroblasts, smooth muscle cells, connective tissue cells, and bone and cartilage cells.

202 PDGF has been shown to increase proliferation of mouse myoblasts and is a key component for

203 the production of CCM (Albrecht \& Tidball, 1997). Isoforms (A, B, C, and D) of PDGF form

204 homo- or heterodimers stabilised by intermolecular disulfide bonding. All four PDGF isoforms

205 feature a highly conserved 100 amino acid GF domain (Breitkopf et al., 2005). Dimerized PDGF 
binds to two cognate tyrosine kinase receptors, platelet-derived growth factor receptor $\alpha$

(PDGFR $\alpha$ ) and PDGFR $\beta$, initiating intracellular signal transduction pathways. Examples of

(Fredriksson, Li, \& Eriksson, 2004).

Commercially available human PDGF-BB is produced recombinantly in E. coli;

211 however, previously published protocols are plagued by low yields, or rely on purification from

212 inclusion bodies under denaturing conditions with the requirement of subsequent refolding to

213 obtain bioactive PDGF-BB (Alexander et al., 1992). To improve upon existing PDGF expression

214 procedures we tested expression of 25 PDGF-BB orthologs (avian, mammalian, reptile, and

215 aquatic) in fusion with N-terminal 6xHis tag and 6xHis-TrxA or 6xHis-DsbA double tags. The

216 6xHis-TEV-PDGF-BB fusions were individually expressed in BL21(DE3)-Gold, Rosetta Gami

217 B, and SHuffle T7 E.coli host strains, but only 1 out of 25 GFs showed soluble expression under

218 these conditions. In contrast, the use of an N-terminal 6xHis-TrxA double fusion resulted in 21

219 out of 25 PDGF-BB produced in a cytoplasmic soluble form (Table S2). We scaled up protein

220 expression and purified 12 of these PDGF-BB orthologues ranging from bovine, bony-tongue

221 fish, cormorant, eagle, cobra, green anole and sea turtle and achieving protein yields ranging

222 from 2-5 mg/L (Figure S1). The 6xHis-TrxA tag was efficiently cleaved from PDGF-BB using

223 TEV protease (Figure 2B); however, in a subset of the orthologs that were purified we observed

224 co-elution of TrxA and PDGF-BB after the second Ni-NTA purification step. Adding a size-

225 exclusion chromatography step was successful in separation of PDGF-BB from TrxA in cases

226 where this tag remained associated with PDGF-BB after TEV protease digestion (Figure S3).

227 The four PDGF-BB orthologues that were not produced as soluble proteins using the

228 pET-TrxA fusion were all sourced from Teleost (ray-finned fish) species. Fusion of these 
orthologues to an N-terminal DsbA tag allowed for their soluble expression and purification.

230 Using this approach, the PDGF-BB from climbing perch (Anabarillus testudineus) was purified

231 at a yield of $7 \mathrm{mg} / \mathrm{L}$. Analysis of all the recombinantly purified PDGF-BB proteins via non-

232 reducing SDS-PAGE confirmed the expected pattern of protein dimerization (Figure S3).

233

234

235

236

237

238

239

240

241

242

243

244

245

246

247

248

249

250

251

\section{Expression and purification strategy for Insulin-like growth factor (IGF-1 and IGF-2)}

IGF-1 and IGF-2 are 70 amino acid disulfide-rich proteins with a similar structure to the peptide hormone insulin (Brown et al., 2008; Vajdos et al., 2001). IGF signalling is central to pathways that control cell growth, maturation, and proliferation. IGF-1 is a major component of FBS and is widely used in cell culture media including in Essential 8 (E8) medium as well as in many different formulations used to promote myogenesis and the development of skeletal muscle (Hakuno \& Takahashi, 2018; McCubrey et al., 1991; Yu et al., 2015). IGF-1 and IGF-2 function on cells by binding to receptor-tyrosine kinases (RTKs), the IGF-1 receptor (IGF1R) and IGF-2 receptor (IGF2R), respectively. IGF-1 and IGF-2 can also bind the insulin receptor, though they do so with lower affinity compared to their interactions with their cognate receptors. IGF-1 and IGF-2 initiate signal transduction through the PI3K/AKT and ERK1/2 signalling pathways resulting in stimulation of cell growth and proliferation (Peruzzi et al., 1999). Commercially available IGF-1 is typically produced in E. coli as inclusion bodies, purified under denaturing conditions and then refolded into the bioactive form. Recent studies have reported that DsbA (Emamipour, Vossoughi, Mahboudi, Golkar, \& Fard-Esfahani, 2019) or 6HFh8 (a six histidine tag followed by the Fh8 peptide from Fasciola hepatica) (Kim et al., 2021) fusion partners promote the soluble expression of mammalian IGF-1 in E. coli.

Based on our success using the TrxA fusion tag to facilitate PDGF-BB expression, we tested the same approach for expression of 15 IGF-1 and IGF-2 orthologs derived from 
mammalian, avian and fish species. This strategy did not result in soluble expression of IGF

253 based on small-scale expression testing (Figure 1A). Next, we assessed the ability of DsbC,

254 DsbA, and SUMO fusion tags to promote soluble expression of IGF in E. coli. Expression of

255 IGF as a DsbC-IGF fusion resulted in significant increases in the production of soluble GF

256 protein (Figures 1A, 2C). With this expression system, all the selected IGF orthologs (human,

257 bovine, fish) were produced primarily as soluble fusion protein (6 IGF-1 and 3 IGF-2), (Figure

258 S1). Yields of DsbC-IGF-1 fusion protein ranged from 6 to $10 \mathrm{mg} / \mathrm{L}$. The DsbA-IGF-2 fusion

259 had similar results in terms of protein solubility, but the overall expression level and yield was

260 generally less than compared to expression of DsbC-IGF-1 fusions. The expression of SUMO-

261 IGF-1 fusions also resulted in production of soluble IGF protein but only in the SHuffle T7 E.

262 coli host strain. TEV protease cleavage of the DsbA/DsbC fusion tags was efficient; however,

263 similar to what we observed for some TrxA-PDGF-BB fusion proteins, the cleaved fusion

264 partner co-eluted with IGF-1 after the second Ni-NTA chromatography step. Anion exchange

265 chromatography (MonoQ) resulted in some degree of separation, but the addition of this step

266 may be problematic at production scales necessary for cellular agriculture applications. To

267 determine if separation of the fusion DsbA/DsbC tag from IGF-1/IGF-2 was a necessary step for

268 bioactivity, we assayed the ability of these fusion GFs to support cell growth and to ascertain if

269 the presence of an N-terminal DsbA/DsbC/SUMO tag had an impact on their functional

270 bioactivity (discussed in sections below).

271 Expression and purification strategy for Transforming growth factor beta 1 (TGF-ß1)

272 TGF- $\beta 1$ belongs to the transforming growth factor superfamily which consists of

273 isoforms TGF- $\beta 1-3$ as well as of other signalling proteins such as myostatin, growth

274 differentiation factor (MSTN or GDF8), and bone morphogenetic factor (BMP) (Poniatowski, 
275 Wojdasiewicz, Gasik, \& Szukiewicz, 2015). TGF- $\beta$ GF are synthesized as a larger precursor

276 protein and then proteolytically cleaved into a 112 amino acid active GF (Derynck et al., 1985).

277 They are rich in cysteine residues and contain multiple intramolecular disulfide bonds

278 participating in formation of a cysteine knot (Hinck et al., 1996). Additionally, disulfide bonding

279 results in the formation of a covalently-linked homodimer that is critical for biological activity.

280 TGF- $\beta 1$ is a key component of the growth medium used for skeletal muscle growth and

281 differentiation and is critical for maintaining the pluripotency of stem cells (Mullen \& Wrana,

282 2017; Scharenberg et al., 2014). For these reasons, it is a major component of growth medium

283 optimized for production of CCM (Boudreault, Tremblay, Pepin, \& Garnier, 2001).

Recombinant production of TGF- $\beta 1$ has traditionally relied on expression using

285 eukaryotic expression systems such as Chinese hamster ovary $(\mathrm{CHO})$ or human embryonic

286 kidney (HEK293) cells, contributing to its high cost and role as a major cost driver in cell culture

287 media (Zou \& Sun, 2004). A recent report detailed successful soluble expression of TGF- $\beta 3$ in $E$.

288 coli in fusion with a TrxA tag (Kuo et al., 2020), but to our knowledge, soluble expression of

289 TGF- $\beta 1$ in E. coli has not been reported. Based on our success in using the TrxA fusion partner

290 to facilitate soluble expression of PDGF-BB, and reports detailing soluble expression of TGF- $\beta 3$

291 using this fusion partner, we attempted to express 10 TGF- $\beta 1$ orthologs (human, bovine, fish,

292 avian) with an N-terminal TrxA fusion. While we did observe expression of a protein species of

293 appropriate size, none of the TrxA-TGF- $\beta 1$ orthologs were expressed as a soluble protein. Next,

294 we tested GB1 tag as the TGF- $\beta 1$ fusion partner, but the results were similar with the expressed

295 protein found only in the insoluble fraction. In contrast, expression of TGF- $\beta 1$ as a DsbA or

296 DsbC fusion protein resulted in several TGF- $\beta 1$ orthologs (human, bovine, fish, avian) expressed

297 in cytoplasmic soluble fraction. Yields of purified DsbC-TGF- $\beta 1$ fusion protein ranged from 4.5 
to $6 \mathrm{mg} / \mathrm{L}$ (Figure 2d). As observed with the IGF-1/IGF-2 GFs, separation of DsbC/DsbA from

299 TGF- $\beta 1$ after TEV-digest required an anion exchange chromatography step.

302 prompted us to try similar strategies for expression of additional GFs that have utility in CCM

303 production. Epidermal growth factor (EGF) is used in differentiation of satellite cells into

304 myotubes and supports the proliferative capacity of mammalian adipose-derived stem cells (Ai et

305 al., 2017). A previous study showed that a GB1 fusion supported soluble expression of human

306 EGF in E. coli (Zheng, 2016). We tested this system on EGF orthologs from fish species and

307 confirmed its ability to promote soluble expression of EGF from Teleost fish species

308 (Supplementary figure 5). We were able to produce soluble GB1-EGF from Betta splendens with 309 a yield of $13.5 \mathrm{mg} / \mathrm{L}$.

311 (Spangenburg \& Booth, 2002). We had success in achieving soluble expression of LIF orthologs

312 from human, bovine, fish, and avian species by using an N-terminal 6xHis-GB1 double fusion

313 partner (Figure S3, Figure S5). Myostatin (GDF8) is a member of the TGF- $\beta$ superfamily and is

314 important in regulating muscle development and skeletal muscle mass. Similar to our results with

315 TGF- $\beta 1$, an N-terminal DsbC fusion facilitated soluble cytoplasmic expression of myostatin

316 (GDF8) orthologues from human and bovine species (Figure S5.). These results further validate

317 our developed methodology for GFs and its application to several GF families relevant to culture

318 media formulation.

319 Validating the functional bioactivity of the recombinantly purified growth factors 
We tested the purified recombinant GFs for their ability to stimulate the proliferation of

321 NIH-3T3 mouse fibroblast cells in a dose-dependent manner using a colorimetric MTT assay.

322 This assay follows the cellular metabolic activity as a measure of viable proliferating cells. To

323 assess the dose-dependent behaviour, serum-starved fibroblast NIH-3T3 cells were individually

324 treated with varying concentrations of each purified GF. All of the recombinantly purified GFs

325 tested demonstrated the ability to support similar and, in some cases higher, cell proliferation

326 than their commercially available mammalian counterparts. We report the cell proliferation

327 capacity of cultures containing each recombinantly produced GF as a fold-change when

328 compared with that of the non-supplemented, serum-starved cells (negative control) under

329 similar experimental conditions (Figure 3). Cells grown on culture medium supplemented with

$33010 \%$ commercial FBS served as the positive control.

The culture medium treated with FGF-2 orthologs derived from bovine (Bos taurus),

332 Atlantic salmon (Salmo salar), Marine medaka (Oryzias melastigma), and Nile tilapia

333 (Oreochromis niloticus), at $10 \mathrm{ng} / \mathrm{mL}$, all showed similar-fold proliferation (8.0 to 8.3-fold for

334 the aforementioned FGF-2 orthologs versus 7.4-fold proliferation with commercial human FGF-

335 2). In the case of FGF-2 orthologs derived from bovine and Atlantic salmon, cells treated with

336 these FGF-2 variants showed 1.5x higher proliferation (6.8-fold and 7.6-fold, respectively), at a

337 much lower ( $1 \mathrm{ng} / \mathrm{mL})$ FGF-2 concentration compared to cells grown in culture medium

338 supplemented with commercial human FGF-2 (5-fold change compared to serum starvation). If

339 this fold-change could be equivalently achieved in bovine myoblast cells or fish cells, it could

340 have a significant impact on the cost incurred for culture media formulation in CCM production.

341 According to our results, the FGF-2 orthologs we tested can accommodate up to a 10X reduction

342 in the amount of FGF-2 added while still promoting similar levels of cell proliferation. 
We observed a similar phenomenon when assaying recombinant FGF-1. FGF-1 orthologs

344 derived from human (Homo sapiens) and bovine (Bos taurus) showed 1.6x higher proliferative

345 capacity when compared to the commercial human FGF-1 at $10 \mathrm{ng} / \mathrm{mL}$. The FGF-1 ortholog

346 derived from chicken (Gallus gallus) showed 3.5-fold proliferation at a concentration of 10

$347 \mathrm{ng} / \mathrm{mL}$ in comparison to 2.5-fold with commercial human FGF-1. At $100 \mathrm{ng} / \mathrm{mL}$, FGF-1 from

348 sheep achieved 1.8x higher proliferative capacity as compared to commercial human FGF-1.

PDGF-BB orthologs derived from bovine (Bos taurus), crested ibis (Nipponia nippon),

350 frog (Xenopus tropicalis), eagle (Haliaeetus albicilla), and green anole (Anolis carolinensis)

351 showed comparatively higher fold-proliferation (3.5- to 4.0-fold) at a much lower concentration

352 of $10 \mathrm{ng} / \mathrm{mL}$ in comparison to commercial PDGF-BB (3.5-fold) proliferation at $50 \mathrm{ng} / \mathrm{mL}$, which

353 is the typical culture medium concentration for PDGF-BB. The ability to produce these PDGF-

354 BB orthologs at a low cost and their potential to sufficiently stimulate proliferation at lower

355 concentrations makes them key candidates for advancing cost reductions in CCM production.

356 All the orthologs of IGFs derived from expression with the different fusion partners

357 (SUMO, DsbC, DsbA) showed similar fold-proliferation as the commercial human IGF-1/IGF-2.

358 Recombinantly purified bovine IGF-1 (DsbC fusion) showed 2.6-fold versus 3.5-fold

359 proliferation for commercial human IGF-1 at $100 \mathrm{ng} / \mathrm{mL}$ concentration when compared to

360 untreated serum-starved cells. Purified IGF-2 orthologs derived from human and bovine both

361 showed similar 2-fold proliferation as the commercial human IGF-2 at a concentration of 100

$362 \mathrm{ng} / \mathrm{mL}$. Interestingly, IGF-2 ortholog from Nile tilapia (Oreochromis niloticus) showed 1.8x

363 higher fold proliferation than the commercial human IGF-2 (3.6-fold and 2-fold respectively at a

364 concentration of $100 \mathrm{ng} / \mathrm{mL})$. 
TGF- $\beta 1$ orthologs from human and bovine both showed 4-fold proliferation as compared to 4.4-fold from commercial human TGF- $\beta 1$ at a concentration of $10 \mathrm{ng} / \mathrm{mL}$. TGF- $\beta 1$ from chicken (Gallus gallus), northern pike (Egretta garzetta), Chinook salmon (Oncorhynchus tshawytcha) and mummichog (Fundulus heteroclitus) showed 3.5-fold, 3.8-fold, 3.6-fold, and 3.4-fold respectively, proliferation at a concentration of $10 \mathrm{ng} / \mathrm{mL}$ when compared with that of untreated cells. Taken together, our results with IGFs and TGF- $\beta 1$ demonstrate that while it is possible to remove the 6xHis-DsbC, DsbA or SUMO fusion partners, the presence of the fusion tags does not interfere with bioactivity of IGF-1/IGF-2 or TGF- $\beta 1$ in cell culture medium.

In addition to measuring the ability of our recombinantly produced GFs to stimulate cell

374 proliferation, we conducted Western blot analysis to observe activation of the downstream cell

375 signalling pathways (ERK, MAPK, Akt) that regulate cellular proliferation. Activation of

376 ERK1/2 through phosphorylation is the key determinant of the cellular response to FGF, PDGF-

377 BB, and IGF growth factor signalling (Zhu, Duchesne, Rudland, \& Fernig, 2010). NIH-3T3 cells 378 treated with the recombinantly purified FGF-2, PDGF-BB, and DsbC-IGF-1/IGF-2 fusion 379 orthologs were analyzed for the detection of phosphorylated ERK1/2 (p-ERK1/2). ERK1/2 380 requires a dual phosphorylation of conserved threonine and tyrosine residues to be activated. Our 381 Western blot results showed bands at 42 and $44 \mathrm{kDa}$, indicative of phosphorylated ERK1/2, thus 382 complementing the results of our cell proliferation assays and validating that our recombinant 383 GFs activate the expected intracellular signalling pathways (Figure 4). Phosphorylation of SMAD proteins is an event that occurs after TGF- $\beta 1$ binds to the 385 TGF $\beta$-receptor at the cell surface, thereby promoting cell proliferation (Schmierer \& Hill, 2007).

386 To confirm that our DsbC-TGF- $\beta 1$ fusion proteins were functionally active, we performed 387 Western blotting against phosphorylated SMAD2 (p-SMAD2). Our results showed that the 
recombinantly purified DsbC-TGF- $\beta 1$ GF were capable of inducing phosphorylation of SMAD2, validating this GF retains the proper signalling functionality even with the DsbC fusion tag. (Figure 4).

\section{Techno-economic analysis of "in-house" growth factor production}

We sought to develop an economic assessment for production of GFs using the methodology in this study. An estimate based on laboratory consumables costs and labour cost

394 showed that we could achieve recombinant production of growth factors for CAD\$ 10.22 per

395 milligram of purified protein (Table 1). A one-time cloning cost incurred for each GF target

396 protein (inclusive of gene synthesis, sequencing primers and PCR) was calculated as

397 approximately CAD\$ 60.00. A more rigorous breakdown for the total cost estimation analysis is 398 presented in the Supplementary section (Table S1).

400 the costs of SFM when sourced from traditional commercial suppliers (Specht, 2020). Using our 401 "in-house" methodology, the overall cost contribution of GFs (FGF-2 and TGF- $\beta 1$ ) to the total

402 cost of SFM can be substantially reduced (Table $2 \mathrm{a}$ ). Tables $2 \mathrm{~b}$ and $2 \mathrm{c}$ summarize a price

403 comparison in the contribution of GF costs, specifically shown for Essential 8 medium

404 demonstrating an 11-fold reduced culture medium cost and a significant reduction in the GF cost 405 contribution to the total media cost from $95 \%$ to $4 \%$. mammalian orthologs that provide comparable or improved bioactivity at lower concentrations

408 also allows for a further reduction in the overall cost of growth medium. Specifically, the ability 
410 reduction as in FGF-2 Atlantic salmon ortholog) can result in even further reductions in media

411 costs.

412 Discussion

413 The presence of mitogenic GFs is a primary requirement for cell culture medium

414 essential for various applications both in academia and bioindustry including the emerging fields

415 of tissue engineering and cellular agriculture. To address the need for cost effective serum-free

416 eukaryotic cell culture medium, we pursued the development of protocols for producing scalable,

417 low-cost recombinantly expressed GFs commonly used in medium compositions. This new data

418 represents a significant advancement for facilitating cost reductions in basic tissue engineering

419 research applications as well as the large-scale applications anticipated for future CCM

420 production.

421 Environmental and ethical concerns surrounding traditional animal agriculture have

422 brought increased focus on the development of alternative sources for dietary protein. CCM

423 represents a promising approach for augmenting existing food production in a land, water, and

424 carbon efficient manner while eliminating the need to harvest from whole animals. Despite its

425 promise, CCM production is currently challenged by the high cost associated with in vitro cell

426 culture, in particular the costs associated with growth medium. While SFM is effective in

427 supporting in vitro cell culture, its cost is often prohibitive for large scale applications, and this is

428 driven primarily by the cost of mitogenic GF components. Addressing this challenge, we have

429 developed an array of expression protocols that facilitate high levels of soluble bioactive GF

430 protein production in E. coli, a tractable host that can be cultured with minimal specialized

431 equipment and in BSL1 laboratory facilities. 
433 chromatography techniques, allowing for the same procedure to be used in preparation of

434 bioactive FGF-1 and FGF-2, IGF-1 and IGF-2, PDGF-BB and TGF- $\beta 1$. The modest demands for

435 laboratory equipment and technical expertise allow for this methodology to be suitable for both

436 academic and industrial settings with minimal equipment and experience in recombinant protein

437 production technology that should facilitate significant reductions in the costs associated with

438 procuring bioactive GFs (Figure 5). Furthermore, we have demonstrated that many of the GFs

439 retain bioactivity as fusion proteins, allowing for purification to proceed via a single

440 chromatography step and without the need for fusion partner cleavage and separation. Despite

441 this observation, we have also outlined and validated strategies for efficient removal of fusion

442 partner tags should it be deemed necessary by end users of these expression systems (Figure S3).

443 To our knowledge, the expression systems we have developed represent the first reported

444 instance of successful expression of soluble and bioactive PDGF-BB and TGF- $\beta 1$ in E. coli

445 expression system.

446 The presented approach allowed for expression of multiple orthologs from each of the GF

447 families, including sequences from mammalian, avian, and Teleost fish origin in accordance with

448 the CCM industry focus on growing skeletal muscle cells belonging to these groups. Though we

449 did not investigate or rationalize why sequence variations amongst GF orthologs dramatically

450 affects their purification and bioactivity characteristics, the ultimate outcome is projected to be a

451 significant cost reduction as decreased amounts of FGF-2, PDGF-BB or TGF $\beta-1$ are suitable for

452 promoting similar fold cell proliferation as the commercial GFs. Further exploration of existing

453 amino acid sequence diversity within these GF families is warranted and may uncover variants

454 that provide improved performance for meeting the unique needs of CCM production. 
In screening GF orthologs for bioactivity, we found several FGF-2 orthologs that induced

456 higher levels of cell proliferation on NIH-3T3 cells compared to commercial mammalian FGF-2.

457 This effect was observed not only at the standard working concentration of FGF-2 (10 ng/mL)

458 but also at reduced concentrations of $0.1-1 \mathrm{ng} / \mathrm{mL}$, raising the possibility that FGF-2 orthologs

459 may provide improved proliferation while using less material. Our functional bioactivity results

460 thus prove a significant cost reduction strategy by using decreased concentrations of FGF-2,

461 PDGF-BB or TGF- $\beta 1$ to promote similar fold cell proliferation as the commercially available

462 GFs.

463 Analysis of the cost of production showed that GFs generated using the protocols we

464 have outlined provide substantial cost savings compared to commercially available alternatives,

465 significantly reducing the cost contribution of GFs to approximately $4 \%$ of the culture medium.

466 Of note, our pricing is based on yields obtained using a bench-scale shake flask approach. It can

467 reasonably be assumed that optimizing growth conditions and transitioning to large-scale

468 bioreactor fermentation could further improve GF yields and facilitate greater cost reductions.

469 The methodology outlined for soluble expression of key GF components is designed to

470 allow for academic laboratories as well as small-scale cellular agriculture start-ups to bring GF

471 production "in-house", thereby allowing for significant cost savings. Most of the expression

472 protocols presented in our study are based on a single IMAC purification and require no in vitro

473 protein refolding steps. While we have documented additional steps that can improve GF purity

474 (e.g., TEV cleavage, size exclusion chromatography, anion-exchange chromatography), in many

475 cases these are optional and may not be required for GFs aimed to be used in cell culture

476 medium. Since the initial purification steps for all these GFs are identical, a standardized 
477 workflow that requires minimal specialized equipment or technical expertise can be adopted to

478 facilitate production of the major cost drivers of SFM (Figure 5).

479 The expression systems we have developed proved to be robust for a variety of mitogenic

480 GFs and represents a key advancement to meeting the increasing demands for these critical

481 components in cell culture media. The set of expression vectors generated in this study also

482 provide a toolset for bacterial expression of other GF families not tested here, and in general, for

483 disulfide-rich proteins more broadly that may have been recalcitrant to bacterial expression

484 system.

$485 \quad$ Limitations of the study

The functional bioactivity of the recombinant growth factors was assayed using fibroblast

487 cell lines. Primary skeletal muscle cells will be a more appropriate model system to test the

488 suitability of their use in cellular agriculture. It was beyond the scope of the work presented to

489 investigate the molecular mechanisms behind the variation in functional activity observed

490 between different growth factor orthologs.

$491 \quad$ Resource Availability

492 Lead Contact

493 Further information and requests for resources should be directed to the corresponding authors,

494 Alexei Savchenko (alexei.savchenko@ucalgary.ca) and Peter Stogios (p.stogios@utoronto.ca)

495 Materials Availability

496 Plasmids used in this study are available upon request.

497 Acknowledgements

498 We thank Dr. Alison McGuigan and members of the McGuigan lab for allowing us to use

499 their cell culture facility and for helpful input on methodology. We also thank Dr. Tom Ben- 
Arye, Senior Scientist, The Good Food Institute (GFI) for scientific input and advice. This work

501 has been funded by a research grant from GFI awarded to Peter J Stogios, a New Harvest

502 Postdoctoral Fellowship Award awarded to Cameron Semper, and a grant from the Animal

503 Advocacy Research Fund awarded to Cameron Semper.

504 Author Contributions

505 Conceptualization, M.V., C.S, P.J.S. and A.S.; Methodology, M.V. and C.S.; Investigation,

506 M.V., C.S., R.D. and N.M.; Writing - Original Draft, M.V. and C.S.; Writing - Review and

507 Editing, M.V., C.S., P.J.S. and A.S.; Funding Acquisition - C.S., P.J.S. and A.S.

508 Materials and Methods

509 Bacterial strains, vector construction: E.coli DH5 $\alpha$ were used for cloning and vector

510 construction. For protein expression, BL21 (DE3) Gold, Shuffle T7 express (NEB), Origami B

511 (DE3), Rosetta Gami B (DE3) (EMD-Millipore) competent cells were used. Commercially used

512 vectors include pMCSG53-His6x-TEV-LIC (MCSG) with LIC cloning sites, pET-His6x-TrxA-

513 TEV -LIC (Addgene 29712), pET- His6x-SUMO-TEV-LIC (Addgene 29711). The vectors

514 pMCSG53-His6x-DsbC-TEV and pMCSG53-His6x-DsbA-TEV were designed "in-house" using

515 the pMCSG53-His6x-TEV-LIC backbone and introducing the DsbC/DsbA gene in frame

516 downstream of His6x tag.

517 All the insert gene sequences were codon optimized for E.coli expression system and synthesized

518 from Twist BioSciences or Codex DNA. The insert genes were cloned into the vector pMCSG53

519 or pET-TrxA or pET-SUMO or pMCSG53-DsbC, pMCSG53-DsbA, coding for a fusion protein

520 with an N-terminal 6x histidine tag cleavable by TEV protease. DNA sequencing in the cloned

521 plasmid was verified at the TCAG DNA sequencing facility, SickKids, Toronto.

522 E.coli competent cells were transformed with the plasmids. $20 \mathrm{~mL}$ of overnight culture (16h

523 approx.) in Luria Bertani (LB) broth was diluted into 1 L of LB or terrific broth (TB) containing 
524 selected antibiotics (100 ug/mL ampicillin) and grown at $37{ }^{\circ} \mathrm{C}$ using the shaker flask approach.

525 Expression was induced with $0.6 \mathrm{mM}$ IPTG at $17{ }^{\circ} \mathrm{C}$ when optical density, OD600 reached 0.8-

5260.9 absorbance units and allowed to grow overnight for 16-18h. The large overnight cell cultures

527 were then collected by centrifugation at $7000 \mathrm{~g}$.

528 Cells were resuspended in binding buffer [pH 7.5, $100 \mathrm{mM}$ HEPES, $500 \mathrm{mM} \mathrm{NaCl}, 5 \mathrm{mM}$

529 imidazole, and 5\% glycerol (v/v)] and lysed. The cell debris was removed by centrifugation at

53020,000 g. Ni-NTA affinity chromatography was used for protein purification. The soluble

531 cytoplasmic fraction containing the GF protein was purified by batch-binding to a Ni-NTA resin,

532 washed with wash buffer [pH 7.5, $100 \mathrm{mM}$ HEPES, $500 \mathrm{mM} \mathrm{NaCl}, 30 \mathrm{mM}$ imidazole, and 5\%

533 glycerol (v/v)], and the protein was eluted with elution buffer [pH 7.5, $100 \mathrm{mM}$ HEPES, 500

$534 \mathrm{mM} \mathrm{NaCl}, 250 \mathrm{mM}$ imidazole, and 5\% glycerol (v/v)]. The His6-tagged protein was then

535 subjected to overnight (16-24h approx.) TEV protease digest using $50 \mu \mathrm{g}$ of "in house" TEV

536 protease per mg of His6x-tagged protein and simultaneously dialyzed overnight against

537 phosphate-buffered saline (PBS), pH 7.4 buffer containing no imidazole. The 6xHis-tag and TEV

538 was removed by binding the protein onto a Ni-NTA column again, whereby the flow through

539 contained the His-tag cleaved protein of interest. For plasmid constructs where the TEV protease

540 digest step was skipped, the final proteins were dialyzed with a minimum of $2 \times 2 \mathrm{~L}$ dilution

541 cycles in (PBS) pH 7.4. For PDGF-BB, the His6x-Trx tag was separated from PDGF-BB using

542 size-exclusion chromatography (Superdex 75 16/60). Fractions containing the PDGF-BB were

543 pooled and concentrated. For TGFB-1 and IGF-1/IGF-2, the His6x-DsbC tag was separated from

544 the GF using anion exchange chromatography (MonoQ 5/5). The purity of all the recombinantly

545 purified proteins were analyzed by SDS-polyacrylamide gel electrophoresis. Non-reducing gel

546 electrophoresis was used to confirm the presence of dimeric state of the proteins. The purified 
547 proteins were also subjected to size exclusion chromatography (Superdex 200 16/60) analysis for

548 determination of their oligomeric state. The proteins were concentrated using a Vivaspin

549 concentrator (GE Healthcare) and passed through a $0.2 \mu \mathrm{m}$ Ultrafree-MC centrifugal filter (EMD

550 Millipore) before storing in aliquots at $-80^{\circ} \mathrm{C}$.

551 Cell culture: NIH 3 T3 mouse fibroblast cell lines (ATCC CRL-1658) were cultured in $100 \times 20$

$552 \mathrm{~mm}$ and $150 \times 25 \mathrm{~mm}$ culture dishes (Falcon Corning cat. no. 353003 and 353025 ) using

553 Dulbecco's modified Eagle's medium containing 4.5 g/L D-glucose, L-glutamine, $110 \mathrm{mg} / \mathrm{L}$

554 sodium pyruvate (DMEM, GIBCO 11995-065) with 10\% heat inactivated newborn calf serum,

555 NBCS (GIBCO 26010-074). Primary pancreatic stellate cells (PSC) were cultured until passage

5568 in DMEM with $10 \%$ heat inactivated fetal bovine serum and penicillin-streptomycin. Cultures

557 were allowed to reach $80 \%$ confluency before passaging them into new media. Briefly, cells

558 were washed twice with phosphate-buffered saline (PBS) without calcium and magnesium,

559 Wisent 311-010-CL, cells detached using Trypsin-0.25\% EDTA (Wisent) and split at a seeding

560 density of 5,000 cells $/ \mathrm{cm}^{2}$. Commercial GFs used in this study included the following: human

561 FGF-1 (PeproTech, cat. no. 100-17A), human FGF-2 (PeproTech, cat. no. 100-18B), human

562 PDGF-BB (PeproTech, cat. no. 100-14B), human IGF-1 (PeproTech, cat. no. 100-11), human

563 IGF-2 (PeproTech, cat. no. 100-12), human TGF- $\beta 1$ (PeproTech, cat. no. 100-21C).

564 Bioactivity MTT assay: NIH-3T3 cells were cultured in 96-well plates at a seeding density of

5656000 cells/well and allowed to attach for $24 \mathrm{~h}$. Cells were subsequently serum starved for $24 \mathrm{~h}$ in

$566 \quad 0.11 \%$ serum medium. GFs were added to individual wells at specific serial dilution

567 concentrations and incubated at $37^{\circ} \mathrm{C}$ with $5 \% \mathrm{CO} 2$. After $48 \mathrm{~h}$, the metabolic activity of viable

568 cells was tested by a colorimetric dimethyl-thiazole-diphenyltetrazolium bromide (MTT) assay

569 (cat. no. M6494, Invitrogen). Briefly, the steps involved aspirating the culture media, washing 
570 the wells with PBS to remove any dead cells, and then adding $100 \mu \mathrm{L}$ of DMEM media

571 containing $10 \mu \mathrm{L}$ MTT (5 mg/mL in sterile PBS) into each well. The 96 well plates were

572 incubated at $37^{\circ} \mathrm{C}$ for $4 \mathrm{~h}$. The media was then aspirated, wells washed with $100 \mu \mathrm{L}$ PBS and the

573 insoluble MTT crystals were solubilized with $50 \mu \mathrm{L}$ of DMSO by incubating in an orbital plate

574 shaker at $700 \mathrm{rpm}, 37^{\circ} \mathrm{C}$ for $20-30 \mathrm{~min}$. Absorbance was recorded immediately on a Tecan plate

575 reader at a wavelength of $540 \mathrm{~nm}$. Three independent experiments were conducted for each set of

576 GFs with different passage number cells. Triplicate readings from each set were averaged and

577 data was presented as mean \pm SD (SD = standard deviation) using Graphpad Prism v5.0.

578 Western blotting: Cells were cultured in 6-well plates at a seeding density of 100,000 cells/well.

579 After $24 \mathrm{~h}$, culture medium in the wells was changed into $0.11 \%$ serum. Cells were serum starved

580 for $24 \mathrm{~h}$ before adding the respective GFs. Cell lysis was done after $45 \mathrm{~min}$ for TGF $\beta 1,24 \mathrm{~h}$ for

581 FGF2, PDGFBB, IGF-1. To extract the total protein, cells were lysed by washing the cells twice

582 in ice-cold PBS, then lysed with 1X RIPA buffer (Cell Signalling Tech. cat. no. 9806), incubated

583 at $4{ }^{\circ} \mathrm{C}$ for $1 \mathrm{~h}$ and centrifuged at $14,000 \mathrm{rpm}$ for $20 \mathrm{~min}$ at $4{ }^{\circ} \mathrm{C}$. The supernatant was mixed with

584 1X SDS-sample buffer containing $\beta$-mercaptoethanol, boiled at $95^{\circ} \mathrm{C}$ for $10 \mathrm{~min}$ and samples

585 loaded onto a 15\% SDS-PAGE protein gel. Proteins were transferred to a nitrocellulose

586 membrane using Trans-Blot apparatus (Bio-Rad). After blocking the membrane with 5\% w/v

587 BSA and $0.1 \% \mathrm{v} / \mathrm{v}$ Tween-20 in TBS (Tris buffered-saline) for $1 \mathrm{~h}$, the membrane was washed 5x

588 with TBST (Tris buffered-saline containing 0.1\% v/v Tween-20). The membrane was incubated

589 with the following primary antibodies overnight at $4{ }^{\circ} \mathrm{C}$ on a rotating shaker: phospho-smad2

590 rabbit mAb (Cell Signalling Tech. cat. no. 3108; 1:1000), smad2 rabbit mAb (Cell Signalling

591 Tech. cat. no. 5339; 1:1000), phospho-p44/42 MAPK ERK1/2 rabbit mAb (Cell Signalling

592 Tech., cat. no. 9101; 1:1000), p44/42 MAPK ERK1/2 rabbit mAb (Cell Signalling Tech., cat. no. 
$5934695 ; 1: 1000)$. The membrane was then incubated with Anti-rabbit IgG, HRP-conjugated

594 secondary antibody (Cell Signalling Tech., cat. no. 7074; 1:2000) with gentle agitation for $1 \mathrm{~h}$ at

595 room temperature. Immunoactivity was detected using Bio-Rad Clarity Western ECL peroxide-

596 enhancer chemiluminescence kit (cat. no. 170-5060). Chemiluminescent bands were visualized

597 with a Chemidoc XRS+ system (BioRad).

598

599

600

601

602

603

604

605

606

607

608

609

610

611

612

613

614

615 


\section{REFERENCES}

Ai, G., Shao, X., Meng, M., Song, L., Qiu, J., Wu, Y., .. . Tong, X. (2017). Epidermal growth factor promotes proliferation and maintains multipotency of continuous cultured adipose stem cells via activating STAT signal pathway in vitro. Medicine (Baltimore), 96(30), e7607. doi:10.1097/MD.0000000000007607

Albrecht, D. E., \& Tidball, J. G. (1997). Platelet-derived growth factor-stimulated secretion of basement membrane proteins by skeletal muscle occurs by tyrosine kinase-dependent and -independent pathways. J Biol Chem, 272(4), 2236-2244. doi:10.1074/jbc.272.4.2236

Alexander, D. M., Hesson, T., Mannarino, A., Cable, M., \& Dalie, B. L. (1992). Isolation and purification of a biologically active human platelet-derived growth factor BB expressed in Escherichia coli. Protein Expr Purif, 3(3), 204-211. doi:10.1016/1046-5928(92)90016$\mathrm{p}$

Benington, L., Rajan, G., Locher, C., \& Lim, L. Y. (2020). Fibroblast Growth Factor 2-A Review of Stabilisation Approaches for Clinical Applications. Pharmaceutics, 12(6). doi:10.3390/pharmaceutics12060508

Bessette, P. H., Aslund, F., Beckwith, J., \& Georgiou, G. (1999). Efficient folding of proteins with multiple disulfide bonds in the Escherichia coli cytoplasm. Proc Natl Acad Sci U S A, 96(24), 13703-13708. doi:10.1073/pnas.96.24.13703

Biancucci, M., Dolores, J. S., Wong, J., Grimshaw, S., Anderson, W. F., Satchell, K. J., \& Kwon, K. (2017). New ligation independent cloning vectors for expression of recombinant proteins with a self-cleaving CPD/6xHis-tag. BMC Biotechnol, 17(1), 1. doi:10.1186/s12896-016-0323-4

Boudreault, P., Tremblay, J. P., Pepin, M. F., \& Garnier, A. (2001). Scale-up of a myoblast culture process. J Biotechnol, 91(1), 63-74. doi:10.1016/s0168-1656(01)00291-7

Breitkopf, K., Roeyen, C., Sawitza, I., Wickert, L., Floege, J., \& Gressner, A. M. (2005). Expression patterns of PDGF-A, -B, -C and -D and the PDGF-receptors alpha and beta in activated rat hepatic stellate cells (HSC). Cytokine, 31(5), 349-357. doi:10.1016/j.cyto.2005.06.005

Brewer, J. R., Mazot, P., \& Soriano, P. (2016). Genetic insights into the mechanisms of Fgf signaling. Genes Dev, 30(7), 751-771. doi:10.1101/gad.277137.115

Brown, J., Delaine, C., Zaccheo, O. J., Siebold, C., Gilbert, R. J., van Boxel, G., . . Jones, E. Y. (2008). Structure and functional analysis of the IGF-II/IGF2R interaction. EMBO J, 27(1), 265-276. doi:10.1038/sj.emboj.7601938

Chen, G., Gulbranson, D. R., Hou, Z., Bolin, J. M., Ruotti, V., Probasco, M. D., . . Thomson, J. A. (2011). Chemically defined conditions for human iPSC derivation and culture. Nat Methods, 8(5), 424-429. doi:10.1038/nmeth.1593

Cheng, Y., \& Patel, D. J. (2004). An efficient system for small protein expression and refolding. Biochem Biophys Res Commun, 317(2), 401-405. doi:10.1016/j.bbrc.2004.03.068

Derynck, R., Jarrett, J. A., Chen, E. Y., Eaton, D. H., Bell, J. R., Assoian, R. K., . . Goeddel, D. V. (1985). Human transforming growth factor-beta complementary DNA sequence and expression in normal and transformed cells. Nature, 316(6030), 701-705. doi:10.1038/316701a0

Emamipour, N., Vossoughi, M., Mahboudi, F., Golkar, M., \& Fard-Esfahani, P. (2019). Soluble expression of IGF1 fused to DsbA in SHuffle T7 strain: optimization of expression and 
purification by Box-Behnken design. Appl Microbiol Biotechnol, 103(8), 3393-3406. doi:10.1007/s00253-019-09719-w

Eschenfeldt, W. H., Makowska-Grzyska, M., Stols, L., Donnelly, M. I., Jedrzejczak, R., \& Joachimiak, A. (2013). New LIC vectors for production of proteins from genes containing rare codons. J Struct Funct Genomics, 14(4), 135-144. doi:10.1007/s10969013-9163-9

Fredriksson, L., Li, H., \& Eriksson, U. (2004). The PDGF family: four gene products form five dimeric isoforms. Cytokine Growth Factor Rev, 15(4), 197-204. doi:10.1016/j.cytogfr.2004.03.007

Gasparian, M. E., Elistratov, P. A., Drize, N. I., Nifontova, I. N., Dolgikh, D. A., \& Kirpichnikov, M. P. (2009). Overexpression in Escherichia coli and purification of human fibroblast growth factor (FGF-2). Biochemistry (Mosc), 74(2), 221-225. doi:10.1134/s000629790902014x

Geistlinger, T., Jhala, R., Krueger, K., Ramesh, B. (2017). United States Patent No.: W. I. P. Organization.

Godfray, H. C. J., Aveyard, P., Garnett, T., Hall, J. W., Key, T. J., Lorimer, J., . . Jebb, S. A. (2018). Meat consumption, health, and the environment. Science, 361(6399). doi:10.1126/science.aam5324

Hakuno, F., \& Takahashi, S. I. (2018). IGF1 receptor signaling pathways. J Mol Endocrinol, 61(1), T69-T86. doi:10.1530/JME-17-0311

Hinck, A. P., Archer, S. J., Qian, S. W., Roberts, A. B., Sporn, M. B., Weatherbee, J. A., . . Torchia, D. A. (1996). Transforming growth factor beta 1: three-dimensional structure in solution and comparison with the X-ray structure of transforming growth factor beta 2 . Biochemistry, 35(26), 8517-8534. doi:10.1021/bi9604946

Kim, Y. S., Lee, H. J., Han, M. H., Yoon, N. K., Kim, Y. C., \& Ahn, J. (2021). Effective production of human growth factors in Escherichia coli by fusing with small protein 6HFh8. Microb Cell Fact, 20(1), 9. doi:10.1186/s12934-020-01502-1

Koledova, Z., Sumbal, J., Rabata, A., de La Bourdonnaye, G., Chaloupkova, R., Hrdlickova, B., . .. Stepankova, V. (2019). Fibroblast Growth Factor 2 Protein Stability Provides Decreased Dependence on Heparin for Induction of FGFR Signaling and Alters ERK Signaling Dynamics. Front Cell Dev Biol, 7, 331. doi:10.3389/fcell.2019.00331

Kuo, H. H., Gao, X., DeKeyser, J. M., Fetterman, K. A., Pinheiro, E. A., Weddle, C. J., .. . Burridge, P. W. (2020). Negligible-Cost and Weekend-Free Chemically Defined Human iPSC Culture. Stem Cell Reports, 14(2), 256-270. doi:10.1016/j.stemcr.2019.12.007

LaVallie, E. R., DiBlasio, E. A., Kovacic, S., Grant, K. L., Schendel, P. F., \& McCoy, J. M. (1993). A thioredoxin gene fusion expression system that circumvents inclusion body formation in the E. coli cytoplasm. Biotechnology (N Y), 11(2), 187-193. doi:10.1038/nbt0293-187

Lobstein, J., Emrich, C. A., Jeans, C., Faulkner, M., Riggs, P., \& Berkmen, M. (2012). SHuffle, a novel Escherichia coli protein expression strain capable of correctly folding disulfide bonded proteins in its cytoplasm. Microb Cell Fact, 11, 56. doi:10.1186/1475-2859-1156

Mattick, C. S., Landis, A. E., Allenby, B. R., \& Genovese, N. J. (2015). Anticipatory Life Cycle Analysis of In Vitro Biomass Cultivation for Cultured Meat Production in the United States. Environ Sci Technol, 49(19), 11941-11949. doi:10.1021/acs.est.5b01614 
McCubrey, J. A., Steelman, L. S., Mayo, M. W., Algate, P. A., Dellow, R. A., \& Kaleko, M. (1991). Growth-promoting effects of insulin-like growth factor-1 (IGF-1) on hematopoietic cells: overexpression of introduced IGF-1 receptor abrogates interleukin-3 dependency of murine factor-dependent cells by a ligand-dependent mechanism. Blood, 78(4), 921-929.

Melzener, L., Verzijden, K. E., Buijs, A. J., Post, M. J., \& Flack, J. E. (2021). Cultured beef: from small biopsy to substantial quantity. J Sci Food Agric, 101(1), 7-14. doi:10.1002/jsfa.10663

Mossahebi-Mohammadi, M., Quan, M., Zhang, J. S., \& Li, X. (2020). FGF Signaling Pathway: A Key Regulator of Stem Cell Pluripotency. Front Cell Dev Biol, 8, 79. doi:10.3389/fcell.2020.00079

Mullen, A. C., \& Wrana, J. L. (2017). TGF-beta Family Signaling in Embryonic and Somatic Stem-Cell Renewal and Differentiation. Cold Spring Harb Perspect Biol, 9(7). doi:10.1101/cshperspect.a022186

Nozach, H., Fruchart-Gaillard, C., Fenaille, F., Beau, F., Ramos, O. H., Douzi, B., . . Dive, V. (2013). High throughput screening identifies disulfide isomerase DsbC as a very efficient partner for recombinant expression of small disulfide-rich proteins in E. coli. Microb Cell Fact, 12, 37. doi:10.1186/1475-2859-12-37

Peruzzi, F., Prisco, M., Dews, M., Salomoni, P., Grassilli, E., Romano, G., . . Baserga, R. (1999). Multiple signaling pathways of the insulin-like growth factor 1 receptor in protection from apoptosis. Mol Cell Biol, 19(10), 7203-7215. doi:10.1128/MCB.19.10.7203

Poniatowski, L. A., Wojdasiewicz, P., Gasik, R., \& Szukiewicz, D. (2015). Transforming growth factor Beta family: insight into the role of growth factors in regulation of fracture healing biology and potential clinical applications. Mediators Inflamm, 2015, 137823. doi:10.1155/2015/137823

Post, M. J., Levenberg, S., Kaplan, D. L., Genovese, N., Fu, J., Bryant, C. J., . . Moutsatsou, P. (2020). Scientific, sustainability and regulatory challenges of cultured meat. Nature Food, 1(7), 403-415. doi:10.1038/s43016-020-0112-z

Rosano, G. L., \& Ceccarelli, E. A. (2014). Recombinant protein expression in Escherichia coli: advances and challenges. Front Microbiol, 5, 172. doi:10.3389/fmicb.2014.00172

Schafer, F., Seip, N., Maertens, B., Block, H., \& Kubicek, J. (2015). Purification of GST-Tagged Proteins. Methods Enzymol, 559, 127-139. doi:10.1016/bs.mie.2014.11.005

Scharenberg, M. A., Pippenger, B. E., Sack, R., Zingg, D., Ferralli, J., Schenk, S., . . ChiquetEhrismann, R. (2014). TGF-beta-induced differentiation into myofibroblasts involves specific regulation of two MKL1 isoforms. J Cell Sci, 127(Pt 5), 1079-1091. doi:10.1242/jcs.142075

Schmierer, B., \& Hill, C. S. (2007). TGFbeta-SMAD signal transduction: molecular specificity and functional flexibility. Nat Rev Mol Cell Biol, 8(12), 970-982. doi:10.1038/nrm2297

Spangenburg, E. E., \& Booth, F. W. (2002). Multiple signaling pathways mediate LIF-induced skeletal muscle satellite cell proliferation. Am J Physiol Cell Physiol, 283(1), C204-211. doi:10.1152/ajpcell.00574.2001

Specht, L. (2020). An analysis of culture medium costs and production volumes for cultivated meat Retrieved from https://gfi.org/wp-content/uploads/2021/01/clean-meat-productionvolume-and-medium-cost.pdf: 
Stout, A. J., Mirliani, A. B., White, E. C., Yuen, J. S. K., \& Kaplan, D. L. (2021). Simple and effective serum-free medium for sustained expansion of bovine satellite cells for cell cultured meat. bioRxiv, 2021.2005.2028.446057. doi:10.1101/2021.05.28.446057

Tripathi, N. K., \& Shrivastava, A. (2019). Recent Developments in Bioprocessing of Recombinant Proteins: Expression Hosts and Process Development. Front Bioeng Biotechnol, 7, 420. doi:10.3389/fbioe.2019.00420

Vajdos, F. F., Ultsch, M., Schaffer, M. L., Deshayes, K. D., Liu, J., Skelton, N. J., \& de Vos, A. M. (2001). Crystal structure of human insulin-like growth factor-1: detergent binding inhibits binding protein interactions. Biochemistry, 40(37), 11022-11029. doi:10.1021/bi0109111

van der Valk, J., Bieback, K., Buta, C., Cochrane, B., Dirks, W. G., Fu, J., . . Gstraunthaler, G. (2018). Fetal Bovine Serum (FBS): Past - Present - Future. ALTEX, 35(1), 99-118. doi:10.14573/altex.1705101

Yang, Z., \& Xiong, H.-R. (2012). Culture Conditions and Types of Growth Media for Mammalian Cells. In L. Ceccherini-Nelli \& B. Matteoli (Eds.), Biomedical Tissue Culture. DOI: 10.5772/52301: IntechOpen.

Yao, T., \& Asayama, Y. (2017). Animal-cell culture media: History, characteristics, and current issues. Reprod Med Biol, 16(2), 99-117. doi:10.1002/rmb2.12024

Yu, M., Wang, H., Xu, Y., Yu, D., Li, D., Liu, X., \& Du, W. (2015). Insulin-like growth factor-1 (IGF-1) promotes myoblast proliferation and skeletal muscle growth of embryonic chickens via the PI3K/Akt signalling pathway. Cell Biol Int, 39(8), 910-922. doi:10.1002/cbin.10466

Zakrzewska, M., Krowarsch, D., Wiedlocha, A., \& Otlewski, J. (2004). Design of fully active FGF-1 variants with increased stability. Protein Eng Des Sel, 17(8), 603-611. doi:10.1093/protein/gzh076

Zheng, W., et al (2016). Expression and purification of human epidermal growth factor (hEGF) fused with GB1. Biotechnology \& Biotechnological Equipment, 30(4), 813-818.

Zhu, H., Duchesne, L., Rudland, P. S., \& Fernig, D. G. (2010). The heparan sulfate co-receptor and the concentration of fibroblast growth factor-2 independently elicit different signalling patterns from the fibroblast growth factor receptor. Cell Commun Signal, 8, 14. doi:10.1186/1478-811X-8-14

Zou, Z., \& Sun, P. D. (2004). Overexpression of human transforming growth factor-beta1 using a recombinant CHO cell expression system. Protein Expr Purif, 37(2), 265-272. doi:10.1016/j.pep.2003.06.001 


\section{Figure Captions}

794 Figure 1. Expression systems for recombinant GF production. (A) Small-scale protein expression screening used to identify the expression vector and host strain combination capable of facilitating cytoplasmic soluble protein expression. The band corresponding to the protein of interest is marked with (*). T - total cell lysate; $\mathrm{S}$ - soluble fraction. (B) Expression vector and host strain combinations for successful expression and purification of soluble, bioactive growth factors. $\left({ }^{\wedge}\right)$ denotes instances where the use of SHuffle T7 Express was required for soluble expression of some orthologs.

Figure 2. Recombinant GF production. Scale up of protein expressions for (A) FGF-2 AND FGF-1 cloned in pMCSG53 vector with N-terminal His6x tag and expressed in BL21(DE3) Gold cells. Targets include F1 (FGF2_Atlantic salmon); F2 (FGF2_Pufferfish); F3 (FGF1_Sheep); F4 (FGF1_Bovine) (B) PDGF-BB expressed in SHuffle T7 express cells. Target shown is P1 (PDGFBB_Cormorant) (C) IGF-1/IGF-2 cloned in pMCSG53-His6x-DsbC/pMCSG53-His6xSUMO and expressed in SHuffle T7 express cells. Targets include (K1) IGF1_Bovine (SUMOHis6x tag); (K2) IGF1_Bovine (DsbC-His6x tag); (K3) IGF1_Goose; (K4) IGF1_Frog; (J1) IGF2_Human; (J2) IGF2_Bovine; (J3) IGF2_Nile tilapia (D) TGF 3 -1 cloned in pMCSG53His6x-DsbC and expressed in SHuffle T7 express cells. Targets shown are TGF $\beta 1$ human (T1); TGF $\beta-1 \_$bovine (T2); TGF $\beta-1$ _chicken (T3); TGF $\beta-1$ _little egret (T4). UC=uncut before TEV digest; $\mathbf{C}=48$ h post - TEV digest; $\mathrm{TEV}$ protease runs at $25 \mathrm{kDa}$ (marked with ${ }^{\wedge}$ ). After the TEV digest and a second Ni-NTA affinity chromatography step, the concentrated, purified FGF2/FGF-1 runs at $15 \mathrm{kDa}$ on an SDS-PAGE (marked with ) shown in (A). PDGF-BB runs at 15 $\mathrm{kDa}$ corresponding to the monomer (marked with $\mathbf{+}$ ) shown in (B). DsbC fusion IGF-1/IGF-2 runs at $35 \mathrm{kDa}$ (marked with $*$ ). IGF1-SUMO runs at $20 \mathrm{kDa}$ (marked with $* *)$, as seen in (C).

Figure 3. Functional bioactivity validation by colorimetric MTT assay. For each growth factor target, cell proliferation is plotted as relative fold-change when compared with that of the untreated serum-starved cells under similar experimental conditions. Absorbance readings at 540 $\mathrm{nm}$ were recorded in six replicates and averaged. Data plotted as mean \pm standard deviation (SD) using Graphpad Prism v5.0. Error bars represent the SD calculated for the replicates. Growth factor concentrations are in $\mathrm{ng} / \mathrm{mL}$.

Figure 4. Western blot analysis of cell extracts from NIH-3T3 cells treated GFs. Cells treated with (A) FGF-2 targets at $10 \mathrm{ng} / \mathrm{mL}$ for $24 \mathrm{~h}$ at $37^{\circ} \mathrm{C}$ (B) PDGF-BB targets at $50 \mathrm{ng} / \mathrm{mL}$ for $24 \mathrm{~h}$ at $37^{\circ} \mathrm{C}$ (C) IGF-1/IGF-2 targets at $100 \mathrm{ng} / \mathrm{mL}$ for $24 \mathrm{~h}$ at $37^{\circ} \mathrm{C}$. All the lysed cell extracts were analyzed for phosphorylated p44/p42 ERK1/2 (p-ERK1/2, 42, $44 \mathrm{kDa}$ ). Negative control (untreated cells) shows no detection of phosphorylated ERK1/2, while all the recombinantly purified FGF-2, PDGF-BB, IGF-1, IGF-2 targets and their commercial counterparts show the presence of bands approximately at $42 \mathrm{kDa}$ and $44 \mathrm{kDa}$ (D) TGF $\beta-1$ targets at $10 \mathrm{ng} / \mathrm{mL}$ for $45 \mathrm{~min}$ at $37^{\circ} \mathrm{C}$ and analyzed for phosphorylated SMAD2 (p-smad2; 60 $\mathrm{kDa}$ ). Negative control (untreated cells) shows no detection of $\mathrm{p}$-smad 2 band while all the recombinantly purified TGF $\beta-1$ targets and commercial TGF $\beta-1$ show the presence of a band at $60 \mathrm{kDa}$. 
838 Figure 5. Protein expression and purification strategy followed for the GF proteins. The

839 flowchart specifically highlights the ease of purification steps by IMAC affinity chromatography,

840 requiring the use of $\mathrm{Ni}-\mathrm{NTA}$ resin and a gravity column.

841

842

843

844

845

846

847

848

849

850

851

852

853

854

855

856

857

858

859

860

861

862

863

864

865

866

867 


\section{(A)}

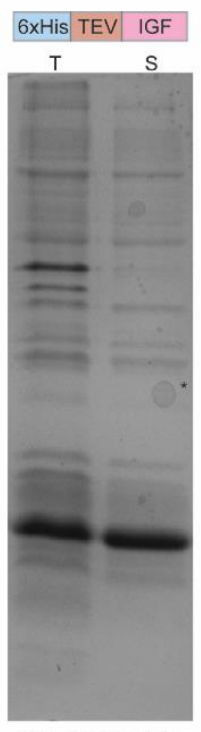

No expression
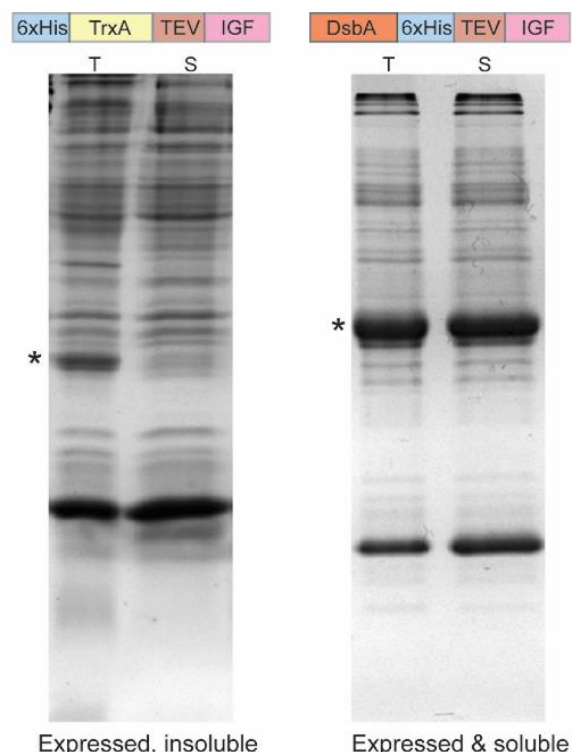

Expressed \& soluble

(B)

Growth Factor FGF-1/FGF-2

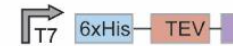

FGF

PDGF-BB

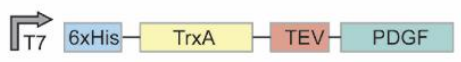

$\sqrt{\mathrm{T} 7}$

DsbA

6xHis- TEV- PDGF

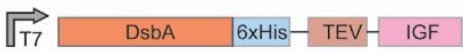

IGF-1/IGF-2

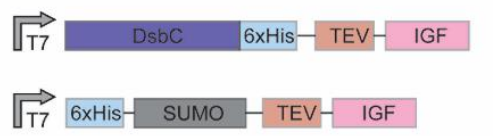

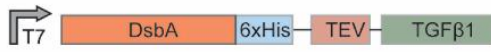

TGF- $\beta 1$

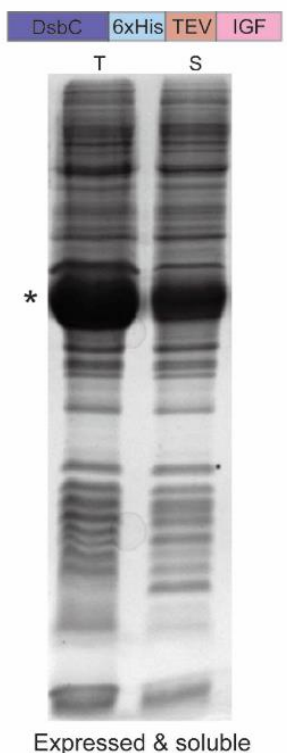

Host strain(s)

BL21-Gold(DE3)

SHuffle T7 Express

SHuffle T7 Express

BL21-Gold(DE3)

BL21-Gold(DE3)^

SHuffle T7 Express

BL21-Gold(DE3)

BL21-Gold(DE3)^

870 Figure 1. Expression systems for recombinant GF production. (A) Small-scale protein expression screening used to identify the expression vector and host strain combination capable of facilitating cytoplasmic soluble protein expression. The band corresponding to the protein of interest is marked with (*). T - total cell lysate; S - soluble fraction. (B) Expression vector and host strain combinations for successful expression and purification of soluble, bioactive growth factors. $\left({ }^{\wedge}\right)$ denotes instances where the use of SHuffle T7 Express was required for soluble expression of some orthologs. 

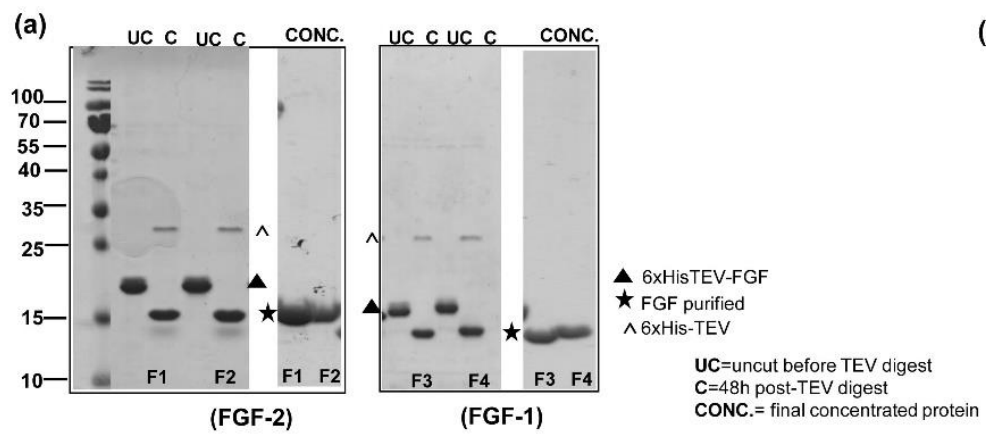

(c)
* 6xHis DsbC-IGF

**6xHis SUMO-IGF

\# 6xHis DsbC-TGF $\beta-1$

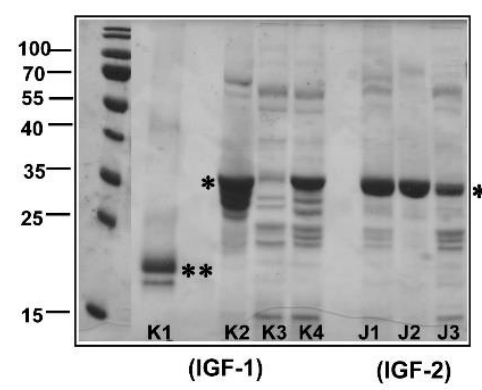
DsbC-TGFß-1 runs at $40 \mathrm{kDa}$ (marked with \#). (b)

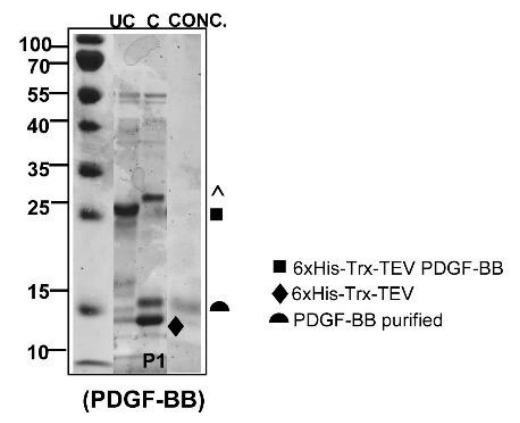

(d)

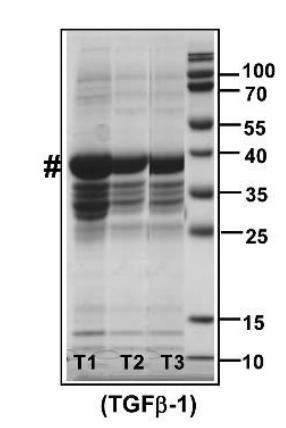

Figure 2. Recombinant GF production. Scale up of protein expressions for (A) FGF-2 AND FGF-1 cloned in pMCSG53 vector with N-terminal His6x tag and expressed in BL21(DE3) Gold cells. Targets include F1 (FGF2_Atlantic salmon); F2 (FGF2_Pufferfish); F3 (FGF1_Sheep); F4 (FGF1_Bovine) (B) PDGF-BB expressed in SHuffle T7 express cells. Target shown is P1 (PDGFBB_Cormorant) (C) IGF-1/IGF-2 cloned in pMCSG53-His6x-DsbC/pMCSG53-His6xSUMO and expressed in SHuffle T7 express cells. Targets include (K1) IGF1_Bovine (SUMOHis6x tag); (K2) IGF1_Bovine (DsbC-His6x tag); (K3) IGF1_Goose; (K4) IGF1_Frog; (J1) IGF2_Human; (J2) IGF2_Bovine; (J3) IGF2_Nile tilapia (D) TGF 3 -1 cloned in pMCSG53His6x-DsbC and expressed in SHuffle T7 express cells. Targets shown are TGF $\beta 1$ human (T1);

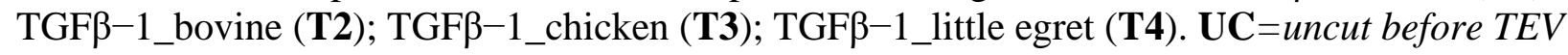
digest; $\mathbf{C}=48$ h post - TEV digest; $\mathrm{TEV}$ protease runs at $25 \mathrm{kDa}$ (marked with ${ }^{\wedge}$ ). After the TEV digest and a second Ni-NTA affinity chromatography step, the concentrated, purified FGF2/FGF-1 runs at $15 \mathrm{kDa}$ on an SDS-PAGE (marked with ) shown in (A). PDGF-BB runs at 15 $\mathrm{kDa}$ corresponding to the monomer (marked with $\boldsymbol{+}$ ) shown in (B). DsbC fusion IGF-1/IGF-2 runs at $35 \mathrm{kDa}$ (marked with $*$ ). IGF1-SUMO runs at $20 \mathrm{kDa}$ (marked with $* *$ ), as seen in (C). 
(a)

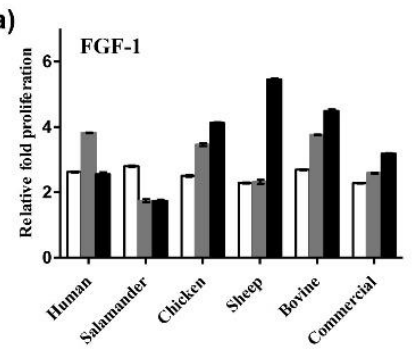

(c)

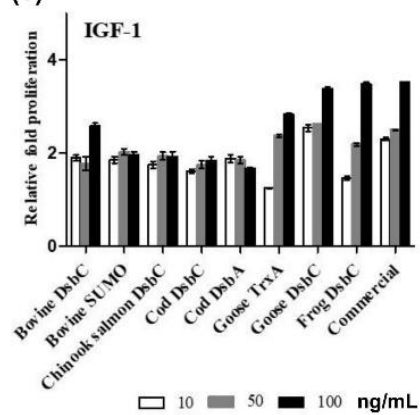

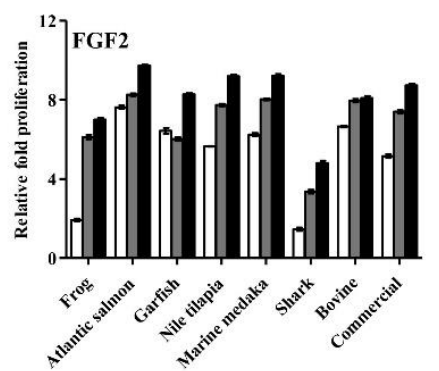

$\square 1 \square 10 \square 100 \mathrm{ng} / \mathrm{mL}$

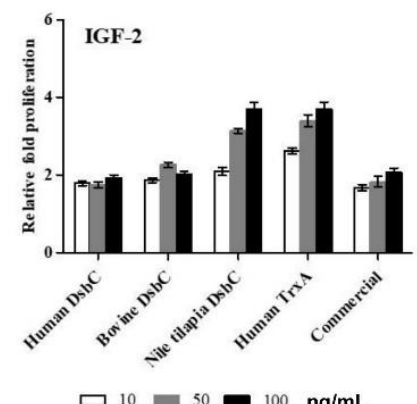

(b)

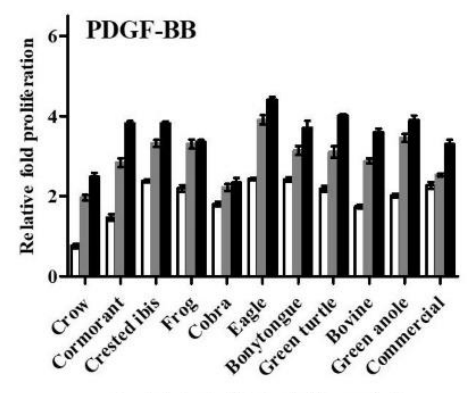

(d)

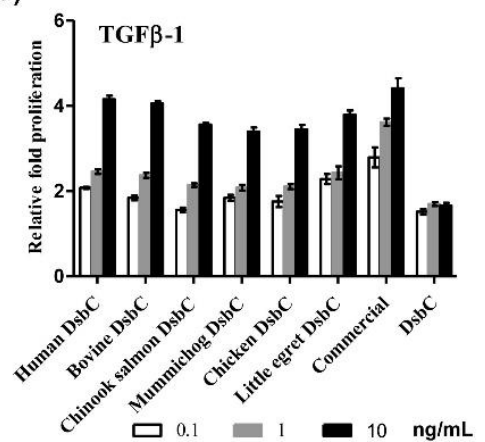

Figure 3. Functional bioactivity validation by colorimetric MTT assay. For each growth factor target, cell proliferation is plotted as relative fold-change when compared with that of the untreated serum-starved cells under similar experimental conditions. Absorbance readings at 540 $\mathrm{nm}$ were recorded in six replicates and averaged. Data plotted as mean \pm standard deviation (SD) using Graphpad Prism v5.0. Error bars represent the SD calculated for the replicates. Growth 
(a) FGF-2

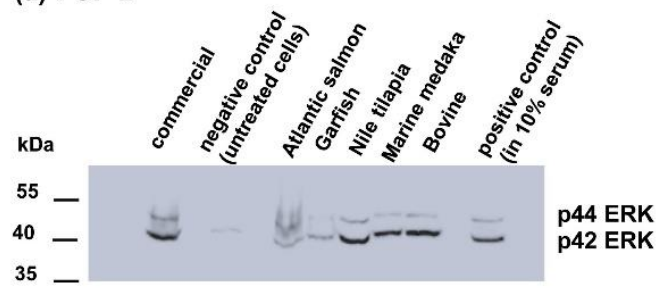

(c) IGF-1/IGF-2

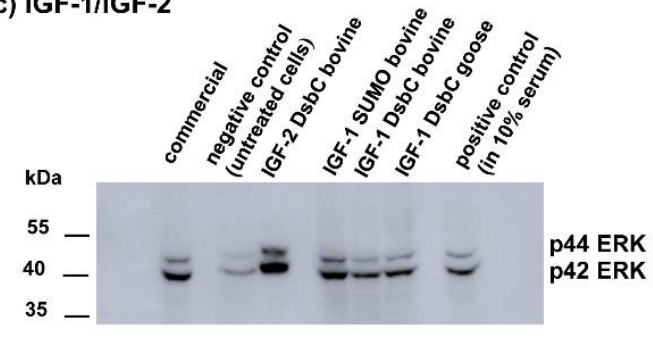

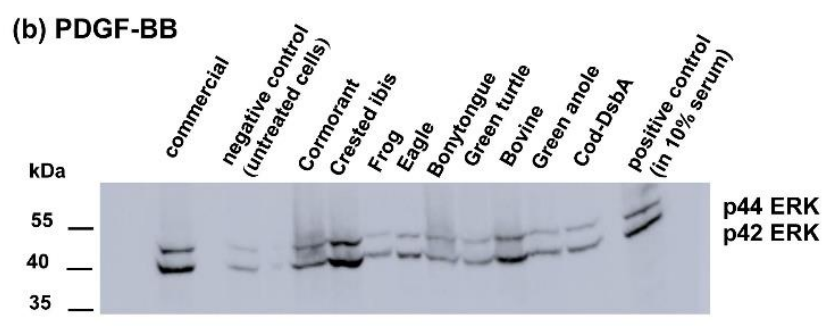

(d) TGFB-1

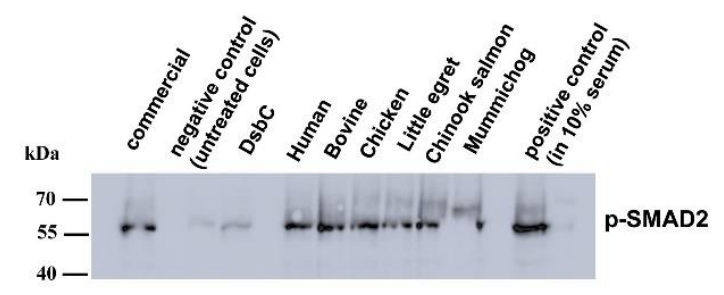

Figure 4. Western blot analysis of cell extracts from NIH-3T3 cells treated GFs. Cells treated with (A) FGF-2 targets at $10 \mathrm{ng} / \mathrm{mL}$ for $24 \mathrm{~h}$ at $37^{\circ} \mathrm{C}(\mathbf{B})$ PDGF-BB targets at $50 \mathrm{ng} / \mathrm{mL}$ for $24 \mathrm{~h}$ at $37^{\circ} \mathrm{C}$ (C) IGF-1/IGF-2 targets at $100 \mathrm{ng} / \mathrm{mL}$ for $24 \mathrm{~h}$ at $37^{\circ} \mathrm{C}$. All the lysed cell extracts were analyzed for phosphorylated p44/p42 ERK1/2 (p-ERK1/2, 42, $44 \mathrm{kDa})$. Negative control (untreated cells) shows no detection of phosphorylated ERK1/2, while all the recombinantly purified FGF-2, PDGF-BB, IGF-1, IGF-2 targets and their commercial counterparts show the presence of bands approximately at $42 \mathrm{kDa}$ and $44 \mathrm{kDa}$ (D) TGF $\beta-1$ targets at $10 \mathrm{ng} / \mathrm{mL}$ for $45 \mathrm{~min}$ at $37^{\circ} \mathrm{C}$ and analyzed for phosphorylated SMAD2 (p-smad2; 60 $\mathrm{kDa})$. Negative control (untreated cells) shows no detection of $\mathrm{p}$-smad 2 band while all the recombinantly purified TGF $\beta-1$ targets and commercial TGF $\beta-1$ show the presence of a band at $60 \mathrm{kDa}$. 

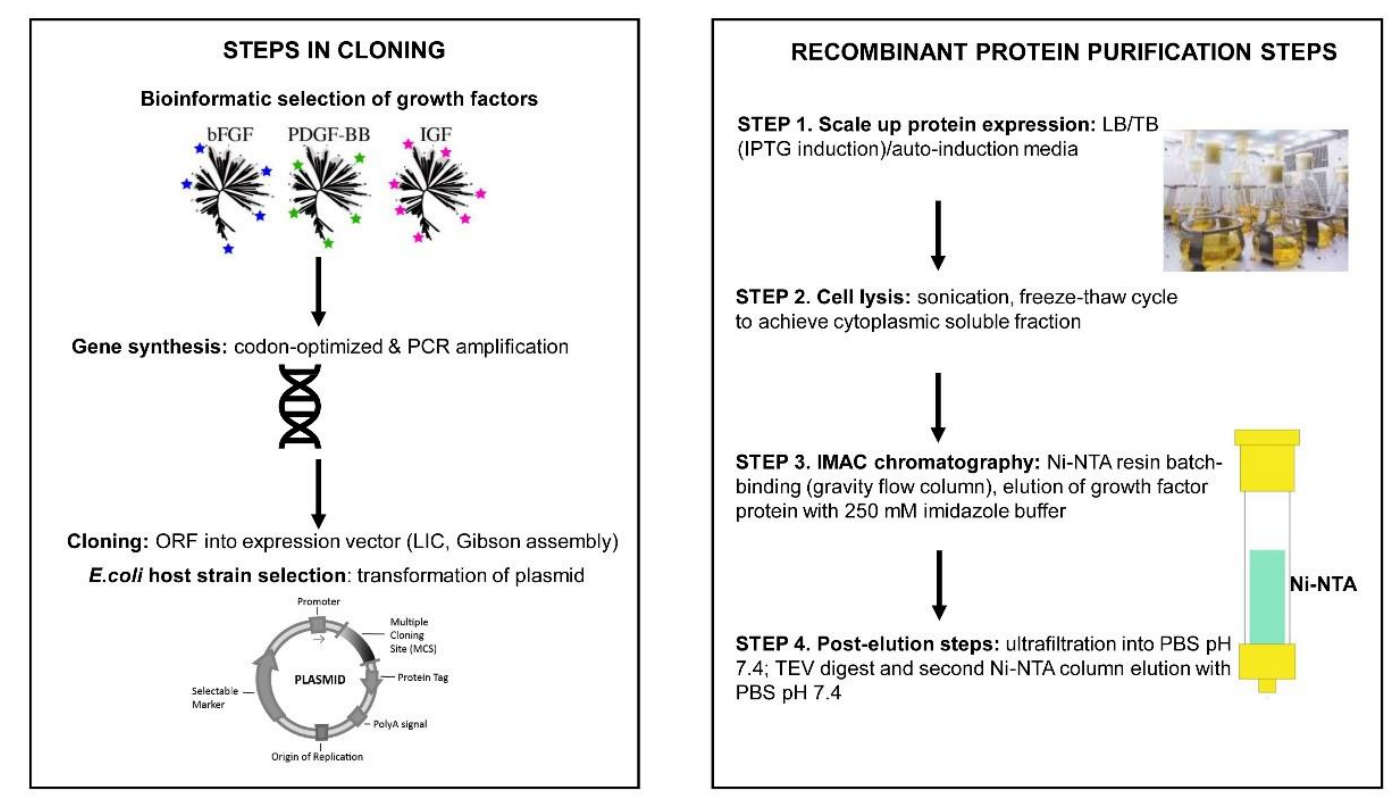

Figure 5. Protein expression and purification strategy followed for the GF proteins. The

948 flowchart specifically highlights the ease of purification steps by IMAC affinity chromatography,

949 requiring the use of Ni-NTA resin and a gravity column. 
Table 1. Production cost of "in house" growth factors: All pricing in CAD and per milligram

966 purified protein. Total production cost of $1225.90 \mathrm{CAD}$ includes all lab consumables and labour 967 costs (see Supplementary Table 1) and estimated for 12 litre production scale of E.coli culture 968 growth. Calculations based on total yield of $120 \mathrm{mg}$ purified protein for all except PDGFBB for which the total yield is $60 \mathrm{mg}$ from a $12 \mathrm{~L}$ culture.

970

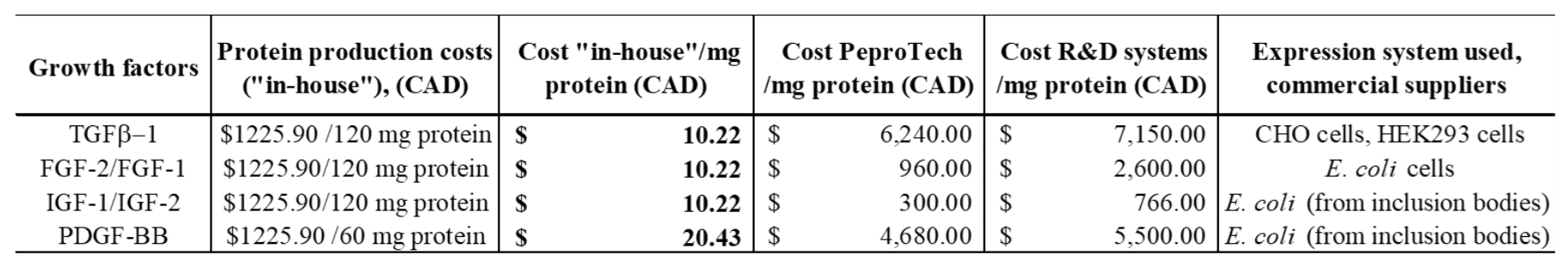


Table 2(a) Pricing comparison of TGF $\beta 1$ and FGF-2 of "in-house" versus commercial (R\&D systems commercial source as reference). Below $\mathrm{mg} / \mathrm{L}$ growth factor calculated based on TGFB1 and FGF-2 composition in Essential 8 media

1008

\begin{tabular}{|c|c|rr|rr|c|}
\hline Growth factors & $\begin{array}{c}\text { Final conc. (in } \\
\text { mg/L) in CCM }\end{array}$ & \multicolumn{2}{|c|}{$\begin{array}{c}\text { Cost/L ("in house"), } \\
\text { CAD }\end{array}$} & $\begin{array}{c}\text { Cost/L (R\&D Systems), } \\
\text { CAD }\end{array}$ & $\begin{array}{c}\text { Pricing comparison, } \\
\text { fold-change }\end{array}$ \\
\hline TGF $\beta 1(10 \mathrm{ng} / \mathrm{mL})$ & 0.01 & $\$$ & 0.10 & $\$$ & 72.00 & $\mathbf{7 2 0 x} \downarrow$ \\
\hline FGF2 $(100 \mathrm{ng} / \mathrm{mL})$ & 0.1 & $\$$ & 1.02 & $\$$ & 260.00 & $\mathbf{2 5 5 x} \downarrow$ \\
\hline
\end{tabular}

\section{1}

1011

1012

1013

Table 2(b) Pricing comparison of total cost of cell culture medium (DMEM basal media and growth factor components). Media pricing was estimated per litre of Essential 8 medium composition. DMEM basal media pricing was derived from thermoFisher vendor (cat. no. $11995065)$ at CAD\$ $15.53 / 500 \mathrm{~mL}$. The cell culture medium cost per litre is 11-fold cheaper using our "in-house" growth factors.

\begin{tabular}{|c|c|c|c|c|}
\hline Growth factors & $\begin{array}{l}\text { Final conc }(\mathrm{mg} / \mathrm{L}) \text { in } \\
\text { cell culture media }\end{array}$ & $\begin{array}{c}\text { Cost/L ("in house"), } \\
\text { CAD }\end{array}$ & $\begin{array}{c}\text { Cost/L (R\&D Systems), } \\
\text { CAD }\end{array}$ & $\begin{array}{l}\text { Pricing compare, } \\
\text { fold-change }\end{array}$ \\
\hline TGF $\beta 1(10 \mathrm{ng} / \mathrm{mL})$ & 0.01 & $\$ \quad 0.10$ & $\$ \quad 72.00$ & $720 \times \downarrow$ \\
\hline \multirow[t]{3}{*}{ FGF2 $(100 \mathrm{ng} / \mathrm{mL})$} & 0.1 & 1.02 & 260.00 & $255 x \downarrow$ \\
\hline & DMEM basal media & 31.06 & 31.06 & N/A \\
\hline & TOTAL COST & 32.18 & 363.06 & \\
\hline
\end{tabular}

Table 2(c) Percentage cost contribution of growth factors (FGF-2 and TGF $\beta$-1) per litre of

\begin{tabular}{c|c}
\hline Vendor & Percentage of GF cost/L \\
\hline "in-house " & 4 \\
PeproTech & 84 \\
R\&D Systems & 92 \\
\hline
\end{tabular}

